\title{
Mitteilungen der DGKJ
}

Monatsschr Kinderheilkd 2021 • 169:582-584

https://doi.org/10.1007/s00112-021-01216-7

(c) Springer Medizin Verlag GmbH, ein Teil von Springer Nature 2021
DEUTSCHE GESELLSCHAFT FÜR KINDER- UND JUGENDMEDIZIN e.V.

Geschäftsstelle der DGKJ

Chausseestr. 128/129, 10115 Berlin

Tel.: +49303087779-0 · Fax: +49303087779-99

E-Mail: info@dgkj.de·Internet:www.dgkj.de

\section{Inhalt}

582 Aufteilung Ihres Mitgliedsbeitrags für 2020

583 Personalia

583 Geburtstage

\section{DEUTSCHE GESELLSCHAFT} FÜR KINDER- UND JUGENDMEDIZIN e.V.

Sie sind noch kein DGKJ-Mitglied?

- Nutzen Sie als Mitglied unserer Fachgesellschaft exklusive Angebote.

- Erhalten Sie die „Monatsschrift Kinderheilkunde“ ohne weitere Kosten.

- Profitieren Sie von den zahlreichen Vorteilen, etwa für Ihre Fortund Weiterbildung.

Weitere Informationen über die Mitgliedschaft in der Deutschen Gesellschaft für Kinder- und Jugendmedizin (DGKJ) sowie den Antrag auf Aufnahme in die DGKJ finden Sie online: www.dgkj.de.

\section{Aufteilung Ihres Mitgliedsbeitrags für 2020}

Sehr geehrte DGKJ-Mitglieder,

wie in jedem Jahr möchten wir Ihnen den Aufteilungsschlüssel für den Mitgliedsbeitrag mitteilen. Der „im Ganzen“ gezahlte DGKJ-Mitgliedsbeitrag muss aus steuerlichen Gründen in einen „echten“ und einen „unechten“ Beitrag aufgeteilt werden. Dieser Verteilungsschlüssel richtet sich nach dem Verhältnis der Kosten der Monatsschrift Kinderheilkunde (inkl. diesbezüglicher Verwaltungskosten) zu den sonstigen originären Vereinskosten und fällt daher in jedem Jahr etwas unterschiedlich aus.

Der Aufteilungsschlüssel für den im Jahr 2020 gezahlten Mitgliedsbeitrag setzt sich wie folgt zusammen:

- Echter Mitgliedsbeitrag 50,52\%,

- unechter Mitgliedsbeitrag 49,48\%.

Nur der Anteil des echten Mitgliedsbeitrags ist eine Zuwendung im Sinne des $\$ 10$ b Einkommenssteuergesetz an eine der in $\$ 5$, Abs. 1 , Nr. 9 des Körperschaftssteuergesetzes bezeichneten Körperschaften, Personenvereinigungen oder Vermögensmassen. Die Differenz aus insgesamt gezahltem Mitgliedsbeitrag und „echtem“ Mitgliedsbeitrag ist als „unechter“ Mitgliedsbeitrag dem Bezug der Monatsschrift Kinderheilkunde zuzuordnen. Dieser Anteil kann ggf. ebenfalls steuerlich abgesetzt werden, allerdings als Betriebsausgabe bzw. Werbungskosten.

Für den Nachweis beim Finanzamt genügen der Kontoauszug, auf dem die Zahlung des Mitgliedsbeitrags vermerkt ist, und dieser Artikel mit dem konkreten Aufteilungsschlüssel.

\section{Prof. Dr. Christian von Schnakenburg}

Schatzmeister der DGKJ 


\section{Personalia}

\section{Nachruf auf Dr. med. Elke Jäger-Roman}

Am Ostersonntag 2021 verstarb plötzlich und unerwartet im Alter von 78 Jahren Frau Dr. Elke Jäger-Roman, eine prägende Persönlichkeit der deutschen Pädiatrie, die sich um die berufsständige Vertretung der deutschen Kinder- und Jugendmedizin und die Professionalisierung der ambulanten kinderärztlichen Versorgung verdient gemacht hat. Wir trauern um eine engagierte Kinderärztin, die sich rückhaltlos für ihre Patienten einsetzte und deren Eltern sich bei ihr immer in sicheren Händen wussten. Unser Mitgefühl gilt ihrer Familie.

Frau Dr. Elke Jäger-Roman wurde am 29.12.1942 in Köln geboren. Nach Kindheit und Jugend in Bonn studierte sie von 1962 bis 1968 Medizin an den Universitäten in Bonn und München. 1963 arbeitete sie 8 Monate in Afghanistan. 1970 erhielt sie die Approbation in Bonn und promovierte. Nach einem halben Jahr chirurgischer Tätigkeit in Südafrika arbeitete sie mit einem Ausbildungsstipendium am Institut für Toxikologie und Embryonal-Pharmakologie der Freien Universität Berlin. Von 1973 bis 1980 ließ sie sich an der Universitätskinderklinik in Berlin, am damaligen Kaiserin-Auguste-Victoria-Haus, zur Fachärztin für Kinder- und Jugendmedizin weiterbilden. Zudem spezialisierte sie sich dort in Kinderrheumatologie. Danach arbeitete sie in der Pädiatrischen Pharmakologie an der Universität von New South Wales in Sydney, Australien.

1982 ließ sie sich in Berlin-Schöneberg als Kinder- und Jugendärztin nieder und betrieb ihre allgemeinpädiatrische Praxis bis 2009. Die ambulante Betreuung von Kindern mit rheumatischen Erkrankungen in ihrer Praxis in West-Berlin zusammen mit Dr. med. Wolfram Singendonk (verstorben ebenfalls 2021) war damals sehr innovativ.

Frau Jäger-Roman war berufspolitisch aktiv im Berufsverband der Kinder- und Jugendärzte (BVKJ) und bekleidete dort hohe Ämter. Es war ihr wichtig, dass die pädiatrische Grundversorgung einen eigenen Versorgungsauftrag hatte und sie forderte daher folgerichtig die Durchführung von mehr Versorgungsforschung, um ein qualitativ hohes Niveau zu erhalten. Daher war sie 2008 maßgeblich beteiligt an der Gründung der Deutschen Gesellschaft für Allgemeine Ambulante Pädiatrie (DGAAP), die dann später in den Konvent für fachliche Zusammenarbeit der DGKJ aufgenommen wurde.

Pädiatrie war für sie immer auch international orientiert, so dass sie den Austausch mit Kinderärztinnen und Kinderärzten in ganz Europa suchte, zunächst in den Vorgängerorganisationen der European

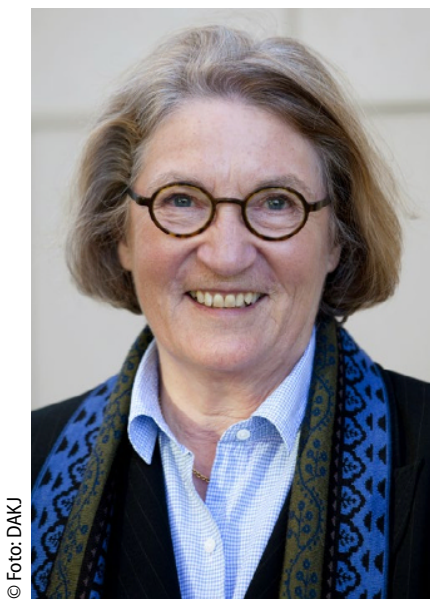

$\Delta$ Dr. Elke Jäger-Roman
Academy of Pediatrics, in der sie seit 2004 deutsche Delegierte war. Später initiierte sie mit anderen primärärztlich interessierten Pädiatern 2009 die Gründung der European Confederation for Primary Care Pediatricians (ECPCP), wurde deren erste Präsidentin und später Ehrenpräsidentin und als „Founding Mother" angesprochen. Noch im März 2021 war sie für die europäischen pädiatrischen Verbände Seniorautorin eines Editorials im Journal of Pediatrics, in dem sie zur kontinuierlichen Durchführung von Prävention und Impfungen unter den Bedingun- gen der SARS-CoV-2-Pandemie aufrief und den ungehinderten und angstfreien Zugang aller Kinder zur Gesundheitsversorgung forderte.

Sie war von 2012-2016 stellvertretende Generalsekretärin der Deutschen Akademie für Kinder- und Jugendmedizin (DAKJ), in die sie vom BVKJ entsandt worden war. Sie war Mitinitiatorin und -autorin der DAKJ-Versorgungsstudie im Jahr 2014. Sie richtete ihr Augenmerk nicht nur auf die medizinischen Versorgungsbedürfnisse, sondern ganz besonders auf die Lebensumstände und Rechte der Kinder und Jugendlichen, in Deutschland und weltweit. Daraus und aus den Erfahrungen bei ihren praktischen Tätigkeiten in Afghanistan und Südafrika resultierte nicht zuletzt ihr unermüdlicher Einsatz für unbegleitete Minderjährige, insbesondere im Zuge des hohen Zustroms von Geflüchteten im Jahr 2015. Zudem initiierte sie die Einrichtung einer Kommission der DAKJ für globale Kindergesundheit.

Hervorzuheben ist in diesem Zusammenhang auch ihr außergewöhnliches Engagement für das Thema Kinderrechte in der deutschen Politik und ihr Bestreben, diese im Grundgesetz zu verankern. Es war ihr unerträglich, dass die Bundesrepublik Deutschland regelmäßige Rügen wegen der mangelhaften Umsetzung der UN-Kinderrechtskonvention scheinbar gelassen hinnahm. So war es ihr wichtig, dass die Pädiatrie als Fach sich auch um die sozioökonomischen Bedingungen kümmert, unter denen Kinder aufwachsen, und dass die Pädiatrie ihre Stimme erhebt, wenn diese Bedingungen der Entwicklung und dem Wohlergehen der Kinder entgegenstehen. Sie trat immer wieder dafür ein, dass die Kinder- und Jugendmedizin in der Öffentlichkeit mit einer Stimme spricht und hat sich deshalb rückhaltlos zur DAKJ bekannt. Die entsprechende Verpflichtung zur Mitarbeit und zum Kompromiss forderte sie von allen Gründungsgesellschaften der DAKJ ein.

Frau Dr. Jäger-Roman hatte ein Auge für verbesserungsbedürftige, für Kinder ungünstige Strukturen und versuchte, die sich daraus ergebenden Probleme im Interesse der Kinder und ihrer Familien zu lösen. Dabei ging sie mit viel mitreißendem Engagement, einem klaren Verstand und großer Zielstrebigkeit immer im Sinne der ihr anvertrauten Kinder und Jugendlichen vor.

Die Klarheit ihrer Aussagen verband sie mit großer Empathie für ihre Gesprächspartner und Kollegen, weshalb auch ihre Kontrahenten ihr immer großen Respekt zollten. Sie war eine immer gut informierte, logisch argumentierende, mitdenkende und warmherzige Gesprächspartnerin, deren Verlust uns schmerzhaft bewusst bleiben wird.

\section{Hans-Iko Huppertz}

\section{Geburtstage}

Die Deutsche Gesellschaft für Kinder- und Jugendmedizin gratuliert herzlich ihren Mitgliedern, die im nächsten Monat einen „besonderen“ Geburtstag feiern:

\section{Jahre und älter werden:}

02.07.1939 | Herr PD Dr. Bernhard Höhmann, CH-Neuenegg

03.07.1939 | Frau Dr. Ebba Müller, Mühlhausen

03.07.1940 | Herr Prof. Dr. Alfried Kohlschütter, Freiburg

04.07.1940 | Herr Prof. Dr. Klaus von Schnakenburg, Bonn

05.07.1939 | Herr Dr. Klaus Hofmann, Lichtenau 
06.07.1939 | Herr Prof. Dr. Christoph Döhlemann, Starnberg 08.07.1934 | Herr Dr. J. August Ermert, Mainz 09.07.1933 | Herr Prof. Dr. Dieter Gmyrek, Radebeul-Wahnsdorf 11.07.1941 | Herr Dr. Wolfgang Meinrenken, Bremen 12.07.1925 | Herr Dr. Hans Kändler, Wismar 14.07.1937 | Herr Dr. Lothar Biskup, Neuss 15.07.1941 | Herr PD Dr. Klaus Heller, Witten 17.07.1933 | Herr Prof. Dr. Hans Carlo Kallfelz, Isernhagen 18.07.1934 | Frau Dr. Ingeborg Lübeck, Hannover 18.07.1937 | Herr Dr. Burkhard Köhler, Stuttgart 18.07.1939 | Frau Dr. Gerlind Domsch, Halle 23.07.1940 | Herr Prof. Dr. Klaus Pistor, Alpen 25.07.1930 | Frau Dr. Erika Bernhardt, Essen 26.07.1932 | Frau Dr. Gisela Schmitz, Düsseldorf 26.07.1940 | Herr PD Dr. Peter Osswald, Gottmadingen 27.07.1940 | Frau Dr. Sigrid von Philipsborn, Regensburg 31.07.1940 | Herr Dr. Volker Siller, Aachen

\section{Jahre werden:}

02.07.1946 | Frau Eva Neuhaus, Bremen 14.07.1946 | Herr Dr. Klaus-Dieter Sparr, Leipzig 20.07.1946 | Herr Dr. Ivo A. Henrichs, Neuburg 25.07.1946 | Frau Dr. Brigitta Becker, Gelsenkirchen 30.07.1946 | Herr Dr. Herrmann Kühne, Neuötting

\section{Jahre werden:}

09.07.1951 | Herr Prof. Dr. Bodo Niggemann, Berlin 11.07.1951 | Herr Dr. Georg Frauendienst-Egger, Pfullingen 11.07.1951 | Herr Dr. Norbert Herrmann, Ansbach 15.07.1951 | Frau Dr. Rene Madeleyn, Filderstadt 16.07.1951 | Frau Dr. Sabine Caspers-Hazay, Hamburg 21.07.1951 | Herr Prof. Dr. Werner Rettwitz-Volk, Dietzenbach

\section{Jahre werden:}

04.07.1956 | Herr Dr. Wolfram Faber, Stuttgart 04.07.1956 | Herr Dr. Thomas Adelt, Bramsche 05.07.1956 | Herr Dr. Matthias Krueger, Klingenberg 08.07.1956 | Herr Prof. Dr. Joachim Kühr, Karlsruhe 08.07.1956 | Herr Dr. Joachim Lemke, Ahrensburg 08.07.1956 | Herr Hanns-Ulrich Leisterer, Zeven 10.07.1956 | Herr Prof. Dr. Jan-Holger Schiffmann, Nürnberg
11.07.1956 | Herr Dr. Alfred Heihoff, Lappersdorf 14.07.1956 | Herr Dr. Ferhat Tezer, Wiesbaden 16.07.1956 | Frau Edith Bieck, Torgau 19.07.1956 | Frau Dr. Martina Oltmann, Bremen 20.07.1956 | Herr Dr. Hartwig Höhre, Heinersreuth 20.07.1956 | Herr Dr. Wolfgang Astfalk, Reutlingen 28.07.1956 | Herr Dr. Christoph Seilacher, Bad Liebenzell 28.07.1956 | Frau Dr. Gabriele Hippke, Stockach 29.07.1956 | Frau Ulrike Schulz, Gardelegen

\section{Jahre werden:}

04.07.1961 | Frau Dr. Sabine Singhof, Königswinter 04.07.1961 | Frau Dr. Christine Hartwig, Stade 04.07.1961 | Herr Dr. Joachim Klumpp, Remagen 05.07.1961 | Herr Dr. Andreas Kästner, Berlin 07.07.1961 | Frau Karen Seel, Berlin 07.07.1961 | Herr Manfred Greve, Wedemark 07.07.1961 | Frau Prof. Dr. Irene Schmid, Germering 09.07.1961 | Frau Dr. Birgit Thalacker, Bremen 10.07.1961 | Herr Dr. Michael Maibohm, Göttingen 12.07.1961 | Frau Dr. Annegret Böhle, Bad Schwartau 13.07.1961 | Frau Barbara Jokiel, Witten

17.07.1961 | Frau Dr. Susanne Gandenberger-Bachem, München 18.07.1961 | Herr PD Dr. Heiner Kentrup, Aachen 21.07.1961 | Herr Dr. Ralf Brangenberg, Traunstein 21.07.1961 | Frau Beate Eger, Pirna 24.07.1961 | Herr Wolfram Krämer, Nieder-Olm 26.07.1961 | Herr Dr. Johannes Buckard, Düsseldorf 26.07.1961 | Frau Dr. Anna-Maria Sonnberg, Berlin 28.07.1961 | Herr Dr. Arnold Köckerling, Biedenkopf 28.07.1961 | Frau Dr. Anne-Katrin Woitke, Märkisch Buchholz

Sollte ein Mitglied nicht wünschen, dass sein Geburtstag in dieser Gratulationsliste erwähnt wird, bitten wir um rechtzeitige Nachricht (4 Monate vor dem Geburtstag) an die

Deutsche Gesellschaft für Kinder- und Jugendmedizin e. V. Chausseestr. 128/129, 10115 Berlin info@dgkj.de 